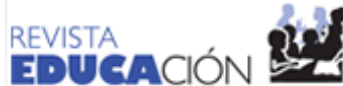

Revista Educación

ISSN: 0379-7082

ISSN: 2215-2644

revedu@gmail.com

Universidad de Costa Rica

Costa Rica

\section{Percepciones del estudiantado argentino de Psicopedagogía en relación a su capacidad empática en la construcción de identidad profesional}

Schlegel, Daiana; Paoloni, Paola Verónica; Gaeta, Martha Leticia

Percepciones del estudiantado argentino de Psicopedagogía en relación a su capacidad empática en la construcción de identidad profesional

Revista Educación, vol. 45, núm. 2, 2021

Universidad de Costa Rica, Costa Rica

Disponible en: https://www.redalyc.org/articulo.oa?id=44066178035

DOI: https://doi.org/10.15517/revedu.v45i1.44467

\section{(c) $(1) \Theta$}

Esta obra está bajo una Licencia Creative Commons Atribución-NoComercial-SinDerivar 3.0 Internacional. 


\title{
Percepciones del estudiantado argentino de Psicopedagogía en relación a su capacidad empática en la construcción de identidad profesional
}

\author{
Perceptions of Psychopedagogy Students in Argentina Regarding their Empathic Capacity in Developing their \\ Professional Identity
}

Daiana Schlegel

Universidad Nacional de Rio Cuarto, Argentina

daiana-schlegel@hotmail.com

iD https://orcid.org/0000-0003-0583-5341

Paola Verónica Paoloni

Universidad Nacional de Rio Cuarto, Argentina

paopaoloni17@hotmail.com

iD https://orcid.org/0000-0002-9384-010X

Martha Leticia Gaeta

Universidad Popular Autónoma del Estado de Puebla,

México

marthaleticia.gaeta@upaep.mx

iD https://orcid.org/0000-0003-1710-217X
DOI: https://doi.org/10.15517/revedu.v45i1.44467 Redalyc: https://www.redalyc.org/articulo.oa? $\mathrm{id}=44066178035$

Recepción: 16 Noviembre 2020

Aprobación: 07 Enero 2021

\section{ReSUMEN:}

La empatía es una de las competencias socio-emocionales más importantes para quienes se desempeñan en el ámbito de la educación. Así, este estudio tiene como objetivo identificar las percepciones que un grupo avanzado de estudiantes de Licenciatura en Psicopedagogía tiene acerca de su capacidad para empatizar. Se empleó un diseño cuantitativo exploratorio. Durante el ciclo lectivo 2019, se trabajó con estudiantes que cursaron Didáctica II en la Carrera de Licenciatura en Psicopedagogía de la Universidad de Río Cuarto (Argentina). Se trató de una muestra incidental constituida por 41 estudiantes. Los datos fueron recabados mediante el Test de Empatía Cognitiva y Afectiva (López-Pérez, Fernández-Pinto y Abad, 2008), en el que se consideraron puntuaciones totales y en relación con sus dimensiones constituyentes: Empatía Cognitiva y Empatía Afectiva. El análisis de los datos se realizó según procedimientos cuantitativos; se empleó el programa PSPP para el procesamiento y análisis de respuestas obtenidas que fueron previamente codificadas. Los resultados destacan fortalezas y debilidades del grupo en estudio respecto de su capacidad empática, el cual obtuvo niveles bajos de empatía -desde una perspectiva global- y puntuaciones medias en la capacidad empática cognitiva y bajas en la capacidad empática afectiva -niveles recomendados teóricamente-. Sin embargo, hay un subgrupo de estudiantes que se percibe con un grado extremadamente bajo de capacidad empática -tanto a nivel global como al interior de cada una de sus escalas-. Los hallazgos obtenidos reflejan la necesidad de pensar en contextos instructivos que ayuden a las futuras personas profesionales a desarrollar empatía como una competencia que les permitirá un mejor desempeño de su rol profesional. Orientado a tal fin, en este trabajo se proponen tres posibles acciones a llevar a cabo en las aulas: promover oportunidades para el autoconocimiento; promover procesos de feedback orientados a destacar cualidades de la persona en situación de aprendizaje, generar contextos ricos en oportunidades para la interacción social.

Palabras Clave: Empatía, Identidad Profesional, Competencias socio-emocionales, Educación Superior, Psicopedagogía.

\section{ABSTRACT:}

Empathy is one of the most important socio-emotional competencies for anyone who works in the field of Education. This study aims to identify perceptions of an advanced group of Psychopedagogy Majors regarding their ability to empathize. The study design was cross-sectional, descriptive and quantitative. During the 2019 school year, we worked with the students who studied Didactics II as part of their Bachelor's Degree studies in Psychopedagogy at the University of Río Cuarto (Argentina). The incidental sample consisted of 41 students. The collected data was based on results from the Cognitive and Affective Empathy Test (López- 
Pérez et al., 2008), which considering total scores and constituent dimensions, cognitive and affective empathy. Data analysis was performed according to quantitative procedures, using the PSPP program for processing and analysis responses that were previously coded. Results highlight strengths and weaknesses of the study group regarding their empathic capacity. The group results reveal low levels of empathy -from a global perspective- and medium scores for their cognitive empathic capacity and low results for affective empathic capacity - all theoretically recommended levels-. However, a subgroup of students was perceived to have an extremely low degree of empathic capacity - both globally and within each of the aforementioned scales. Findings reflect the need to provide instructional contexts toa aid future teachers in to developing greater empathy - a skill that will benefit their professional career. Three possible actions to be carried out in the classrooms were proposed to this end: promoting opportunities for self-knowledge; promoting feedback aimed at highlighting qualities of students, providing more opportunities for social interaction.

KEYWORDS: Empathy, Professional Identity, Socio-emotional Competence, Higher Education, Psychopedagogy.

\section{INTRODUCCIÓN}

Hallazgos obtenidos en el marco de estudios previos, ponen de manifiesto la importancia que supone considerar las percepciones y valoraciones que el estudiantado ha construido acerca de sí mismo para los aprendizajes y el rendimiento obtenido (Paoloni, 2015; Paoloni, Chiecher y Martín, 2015; Ryan y Deci, 2000; Volet, 2011).

Entre los aspectos personales del alumnado que influyen en sus aprendizajes, se ha considerado particularmente la motivación (Chiecher, Paoloni y Ficco, 2014; Hernández, Martín, Lorite y Granados, 2018; Järvelä y Niemivirta, 2001; Sorolla, Alvarez y Penna, 2019), la dinámica emocional (Medina, 2020; Oyarzún-Yáñez y Valdés-León, 2020; Pacheco-Hernández, 2016; Paoloni y Rinaudo, 2015; Pintrich y Schunk, 1996) y el autoconcepto (Bongy Skaalvik, 2003; Marsh, 2014; Paoloni, 2014; Sevilla-Santo, MartínPavón, Sunza-Chan y Druet-Domínguez, 2021). Sin embargo, aunque los estudios efectuados han logrado avances interesantes en el conocimiento del rol que desempeñan estas variables personales, es muy poco lo que se conoce aún sobre el papel que desempeñan las percepciones del estudiantado acerca de sus competencias socio-emocionales, principalmente acerca de la empatía en la construcción del rol profesional.

$\mathrm{Al}$ respecto, uno de los estudios más actuales que se consultó y que -entre otros aspectos- considera cuán eficaces se percibe el alumnado del nivel terciario de Carreras de Profesorado para empatizar con los demás (Aristulle y Paoloni, 2019), indica que el $62 \%$ de la población participante reconoce tener muy desarrollada esta competencia, mientras que el $38 \%$ restante manifiesta tener poco o nada desarrolla esta habilidad básica para "discernir y responder adecuadamente al humor, el temperamento, las motivaciones y los deseos de los demás” (Goleman, 2007, p. 60). Como lo indican las autoras, en este trabajo lo que inquieta es el alto porcentaje de estudiantes - casi un $40 \%$ - que admite haber desarrollado poca o nula capacidad para empatizar. Las respuestas proporcionadas por este grupo de estudiantes promueven una reflexión acerca de que la educación es, ante todo, un problema político que exige pensar qué tipo de sociedad se aspira construir. Si se espera que la formación profesional contribuya a fortalecer la ciudadanía, la democracia, la convivencia pacífica, la búsqueda del bien común; se espera que se facilite en el estudiantado el desarrollo de competencias ciudadanas congruentes con formas pacíficas de asociación y de resolución de conflictos, de legalidad e institucionalidad democráticas y convivir en el respeto a la diversidad y los derechos humanos de todas las personas. Entonces, la capacidad para empatizar es clave y se torna importante indagar acerca de ella y de promover su desarrollo en la formación de profesionales de la educación -tal y como se plantea en el estudio puesto a consideración-.

Por su parte, entre los estudios que se enfocan específicamente en la identidad profesional y, en particular, en la percepción de la capacidad empática, se encuentran algunos trabajos que fueron realizados con profesionales en ejercicio. Se trata de estudios a gran escala llevados a cabo en el ámbito de la Medicina (Ávila-Alarcón, Carrasco, Osorio, Calzadilla y Díaz, 2020; Czapski y Lassi, 2020; Guilera, Batalla y SolerGonzález, 2020; Huarcaya-Victoria, Cano-Uría, Villanueva-Ruska y de la Cruz-Oré, 2019). Entre otros 
hallazgos, llama la atención los niveles relativamente bajos del estudiantado de medicina, principalmente en las dimensiones denominadas cuidado con compasión y habilidad para entender a otras personas, que indicarían dificultades a la hora de expresar compasión con sus pacientes o reconocer las emociones de alguien más. Al mismo tiempo, estos trabajos muestran la necesidad y el valor de generar intervenciones concretas para enriquecer los niveles de empatía de la población estudiantil. Al respecto, las personas autoras entienden que, por lo general, los planes de estudios en medicina se caracterizan por centrarse en conocimientos teóricos y técnicos, por lo que descuidan el aprendizaje de competencias vinculadas a la capacidad empática y otros aspectos emocionales.

En definitiva, tal y como lo muestran los resultados presentados, la empatía en la formación profesional se torna una competencia clave para pensar en una sociedad más equitativa y democrática. Para este trabajo se parte del supuesto de que conocer más acerca de las percepciones de estudiantes sobre su empatía -y generar instancias de reflexión respecto de los resultados obtenidos-, brindaría herramientas conceptuales y metodológicas más sensibles, capaces de promover mejoras en propuestas de enseñanza orientadas a enriquecer los procesos de construcción del rol profesional.

Según lo planteado, el objetivo general de esta investigación se orienta a identificar las percepciones que un grupo de estudiantes en nivel avanzado de psicopedagogía tienen acerca de su competencia para empatizar. Se considera que los resultados obtenidos pueden aportar elementos de juicio capaces de ampliar la reflexión y el debate sobre contextos de aprendizaje que contribuyan a desarrollar esta competencia en particular desde la formación de grado.

\section{CONSIDERACIONES CONCEPTUALES}

\subsection{La formación profesional en la licenciatura en psicopedagogía: consideraciones acerca de la identidad profesional}

Una revisión de antecedentes sugiere que, en las últimas décadas, se advierte un paulatino desplazamiento de visiones que tendieron a dividir los vínculos existentes entre persona y situación hacia perspectivas más integrales, las cuales atienden a la complejidad del actuar de la persona en situación como clave para comprender el compromiso asumido con los aprendizajes (Rinaudo, 2014). En este sentido, junto a los aportes de las teorías socio-constructivistas en el campo de la psicología educacional, en la actualidad se han logrado perspectivas más integrales de la educación, donde se ve al estudiantado como protagonista. Un paradigma desde el cual se propone:

(...) el desarrollo de competencias para la formación de ciudadanos autónomos, capaces de emitir juicios y tomar decisiones responsables, con base en el conocimiento y razonamiento, que les permita aprender de manera continua y resolver problemas más allá del contexto escolar, y en diferentes etapas de su vida (Gaeta, 2014, p. 33).

Con base en las perspectivas mencionadas, uno de los propósitos para la formación profesional en psicopedagogía es ofrecer una serie de situaciones didácticas que permitan al estudiantado interpretar procesos de enseñanza en sus diversas dimensiones, y sus relaciones con los procesos de aprendizaje a partir de la comprensión profunda de diferentes contextos en el trabajo interdisciplinario. Además, se busca ampliar los campos de observación y que el estudiantado logre delimitar nuevos contextos de acción educativa que se constituyan en ámbitos de trabajo profesional y de acción social. En pocas palabras, se espera ofrecer lo que, para Rinaudo (2014), constituyen contextos de aprendizaje poderosos en sus posibilidades para promover la conformación de identidades profesionales integrales.

De acuerdo con Rinaudo (2014), estos contextos se caracterizan por promover en el alumnado la puesta en práctica de la capacidad crítica y reflexiva como sujetos activos en su proceso de aprendizaje y, sobre todo, como futuras personas profesionales de la educación. Específicamente, la autora plantea, como uno de 
los principales objetivos de la tarea educativa, el hacer que la población estudiantil tome el rol de "agentes responsables de sus actuaciones en el campo personal, laboral y social. El desarrollo de esta capacidad de hacerse cargo, de sentirse capaz de pensar y actuar en consonancia con metas personales y sociales" (Rinaudo, 2014, p. 14). Lo que se intenta es brindar oportunidades para reflexionar sobre su futuro rol profesional, avanzar en la delimitación de metas personales de mediano y largo plazo, enriquecer su base de conocimientos y habilidades intelectuales vinculadas al campo de estudio, atravesados por la capacidad de desplegar sus competencias socio-emocionales en diferentes ámbitos, aspecto en el que se profundizará en el apartado siguiente.

Desde perspectivas socioculturales actuales, se entiende por identidad a las:

(...) representaciones de sí mismas que las personas construyen a lo largo de sus vidas, dentro de los contextos socioculturales de los que forman parte. Su construcción implica un proceso de negociaciones personales e interpersonales que tienen lugar en el marco de sistemas de significados, prácticas y estructuras que regulan su continuo desarrollo (Rinaudo y Paoloni, 2015, pp. 78-79).

La construcción de la identidad -como proceso que se despliega a lo largo de toda la vida- se logra entonces de manera conjunta con la otredad, en el seno de diversas influencias culturales y prácticas socioculturales (Paoloni, Rinaudo, Chiecher y Martín, 2017). Se trata de un proceso complejo, dinámico y sostenido en el tiempo, que implica un modo de entender las relaciones con otras personas en función de roles laborales.

Por su parte, la identidad profesional -como una particularización de la identidad general- es definida como la imagen que tiene de sí misma la persona en relación con el desarrollo de su profesión, y comprende aspectos relacionados al ejercicio de la profesión, tanto de forma independiente como dentro de una organización (Elliot, 1975). Aisenson et al. (2005), por su parte, entienden a las representaciones sociales acerca de una profesión como una forma de saber práctico que vincula a un sujeto con un objeto social. "Se trata de un conjunto de elementos informativos, actitudes, creencias, valores, opiniones, imágenes, etc., que están organizados como un saber que dice algo sobre algún objeto social” (Aisenson et al., 2005, p. 36). Específicamente, el saber práctico hace referencia a la experiencia a partir de la cual es producido, a los contextos y condiciones en los cuales se manifiesta y, sobre todo, al hecho de que las representaciones se constituyen en una guía para actuar en el mundo y con los demás. Lasky (2005) agrega que se trata de una construcción subjetiva, que concentra el compromiso personal, las creencias y valores asociados a la profesión que son definidos culturalmente.

Por lo expuesto hasta aquí, se comprende a cabalidad que las identidades profesionales son integrales cuando en su construcción se atiende no sólo a conocimientos conceptuales y procedimentales, sino que también se integran los aprendizajes acerca de los modos de ser y estar individual y con las demás personas en el ejercicio del rol profesional. Son identidades que trascienden los conocimientos técnicos, la aptitud intelectual o las habilidades cognitivas para avanzar decididamente y de modo complementario hacia el desarrollo de diversas competencias emocionales y relacionales (Paoloni, 2018; Rinaudo, 2019).

Actualmente, se apunta desde los sistemas educativos, y principalmente desde la Universidad Nacional de Río Cuarto, a una formación integral. Se busca formar personas con capacidad de escucha, diálogo y compromiso, así como con pensamiento crítico y capacidad empática; es decir, se busca formar personas profesionales con competencias intrapersonales -que les permita

comprenderse a sí mismas como sujetos de cambio- y competencias interpersonales que amplíen sus posibilidades para comprender a las demás personas y comprometerse con las necesidades de su entorno (Colby, Ehrlich, Beaumont y Stephens, 2003; Gaeta y Martínez-Otero, 2017; Vallaeys, De la Cruz y Sasia, 2009). En el presente estudio se focaliza la atención en una de estas competencias interpersonales: la empatía. 


\subsection{Algunas consideraciones acerca de la empatía como competencia clave para el desempeño profesional}

Los seres humanos por naturaleza hacen que el reconocimiento y la comprensión del estado de los demás tome especial relevancia en relación con el resto de los aspectos de la vida (Richaud, 2017). Esto es denominado empatía, competencia clave para la vida en sociedad.

Es frecuente que la empatía se defina de manera coloquial como colocarse en los zapatos del otro. Pero al ahondar en su definición, se halla una gran diversidad de posturas y modelos a lo largo de la historia, los cuales se han ido centrando en diferentes aspectos. Autores como Hogan (1969) la consideran una capacidad cognitiva clave para comprender lo que piensan las demás personas; mientras que otros autores y autoras destacan el componente afectivo y la describen como una respuesta afectiva a las emociones ajenas (Bryant, 1982; Hoffman, 1982; Mehrabian y Epstein, 1972).

Por la década de 1980 se fue generando una mirada integral de ambos componentes -cognitivo y afectivo- (Davis, 1983), que definen la empatía como un constructo multidimensional. Específicamente, fue conceptualizada como un conjunto de construcciones vinculadas con las respuestas de una persona en relación con la experiencia de otro; es decir, la integración de los procesos de ponerse en el lugar de la otra persona y las respuestas afectivas y no afectivas (Davis, 1983, 1996). Desde esta perspectiva, el presente estudio acuerda en definir la empatía como la competencia por medio de la cual las personas pueden conocer con más certeza cómo se sienten las demás, qué es lo que están pensando, comprender sus intenciones, predecir sus comportamientos y entender sus emociones (Baron-Cohen y Wheelwright, 2004).

Desde este modelo integral de empatía, Decety y Jackson (2004) plantean tres componentes que interactúan dinámicamente, a saber: la emoción compartida entre quien observa y quien observado; la autoconciencia y conciencia del otro, en referencia a la diferenciación entre el self y el otro; y la flexibilidad cognitiva para adoptar la perspectiva ajena. A la propuesta de Decety y Jackson (2004), Gerdes y Segal (2009) suman el concepto de acción empática o conducta prosocial. Este nuevo componente se refiere a la decisión consciente de emprender una acción empática, es decir, acciones orientadas a buscar el bien de las demás personas. Ambas propuestas contemplan la dimensión afectiva - preocupación empática- y la dimensión cognitiva -toma de perspectiva- (Baron-Cohen y Wheelwright, 2004; Zorza y Beccaría, 2014).

\subsubsection{La empatía en el marco de diversos modelos de competencias socioemocionales}

Las competencias socio-emocionales se definen como "el conjunto de conocimientos, capacidades, habilidades y actitudes necesarias para realizar actividades diversas con un cierto nivel de calidad y eficacia" (Bisquerra, 2003, p. 21). Dicho concepto integra el saber, saber hacer y saber ser, por lo cual cada competencia, habilidad o capacidad es aprendida y susceptible de poder ser desarrollada (Repetto-Talavera y Pérez-González, 2007).

En el desarrollo conceptual acerca de las competencias socio-emocionales, Paoloni (2019) advierte que, a lo largo de los años, se observa una diversidad de modelos conceptuales. Por ejemplo, en los desarrollos sobre inteligencias múltiples propuestos por Howard Gardner (1998) -particularmente lo que atañe a la inteligencia interpersonal e inteligencia intrapersonal- o en los modelos sobre Inteligencia Emocional propuestos por Mayer y Salovey (1997), Daniel Goleman (2000; 2013), Reuven Bar-On (2000; 2006; 2012), etc., la empatía ocupa un lugar fundamental.

En definitiva, la empatía es una competencia socio-emocional sustancial para diferentes ámbitos del desarrollo personal y social. Concretamente, en el ámbito educativo, diferentes investigaciones han encontrado relaciones significativas entre la empatía y la conducta prosocial, el establecimiento de vínculos sociales de calidad y el altruismo (Martínez-Otero, 2017; Vital, Gaeta y Martínez-Otero, 2017). A nivel 
más general se encontraron relaciones entre la empatía y la conducta moral (Hoffman, 2001) y la tendencia afiliativa (Mehrabian, 1976) e inversamente proporcional con la agresividad (Miller y Eisenberg, 1988).

Tal como se destacó en el apartado anterior, la empatía es una de las competencias destacadas en gran parte de los propósitos de la formación académica del profesional en educación. Sin embargo, en Argentina es necesario ampliar la base de estudios que consideran a la empatía en la formación de identidades profesionales integrales, sobre todo en el ámbito de la psicopedagogía, debido a que no se han hallado estudios en la temática .

\section{Consideraciones Metodológicas}

\subsection{Participantes y contexto donde se llevó a cabo la investigación}

La investigación tuvo lugar en la Universidad Nacional de Río Cuarto (UNRC), provincia de Córdoba, República Argentina. Se empleó un diseño cuantitativo exploratorio [1]. Se trabajó con 41 estudiantes de la Facultad de Ciencias Humanas de la UNRC; esto es, el total de estudiantes de la Licenciatura en Psicopedagogía que cursaron en el año 2019 Didáctica II -asignatura ubicada dentro del programa de la Carrera correspondiente al 4to año-. Se trató de una muestra incidental definida por el criterio de accesibilidad a los datos. La totalidad del grupo con el que se trabajó fue de género femenino, con edades que oscilan entre 20 y 28 años. Su participación fue voluntaria y anónima; se destacó la confidencialidad de los datos proporcionados en el marco del estudio llevado a cabo. El trabajo realizado no contradice la Ley 25.326 de protección de los datos personales (Normativa nacional, Argentina), no se consideran datos sensibles para la investigación y los datos empleados se recabaron con contentamiento informado, se garantizó el anonimato de personas participantes y se resguardó la confidencialidad de los datos proporcionados.

\subsection{Instrumento y procedimientos considerados en el estudio}

Para explorar la empatía que el estudiantado reconoce en sí, se empleó el Test de Empatía Cognitiva y Afectiva -TECA- de López-Pérez et al. (2008) ${ }^{[2]}$. Se trata de un instrumento de autoinforme compuesto por 33 ítems o frases que listan sentimientos y pensamientos en diferentes situaciones percibidos por el estudiantado. Cada ítem del TECA se puntúa según una escala de 5 puntos -tipo Likert- que va de 1 Totalmente en desacuerdo a 5 Totalmente de acuerdo.

La finalidad de este test es proporcionar datos que permitan una apreciación más objetiva de la capacidad empática de la persona desde una perspectiva global que integra, a su vez, una dimensión cognitiva comprensión del estado interno de otra persona- y otra afectiva -reacción emocional de la persona al observar experiencias de otras personas- (Ruiz-Baca, 2016). Se consideran cuatro dimensiones específicas, en cada dimensión, para la valoración de la empatía: adopción de perspectivas y comprensión emocional integran la dimensión cognitiva; estrés empático y alegría empática conforman la dimensión afectiva. A continuación, se expone a qué hace referencia cada una de estas sub dimensiones según las personas autoras del instrumento:

a) Adopción de perspectivas. Hace referencia a la capacidad intelectual o imaginativa de ponerse en el lugar de otra persona. Está integrada por un total de ocho ítems. Por ejemplo: Antes de tomar una decisión intento tener en cuenta todos los puntos de vista (item6); Intento ponerme en el lugar de las demás personas para saber cómo actuarán (item15).

b) Comprensión emocional. Alude a la capacidad de reconocer y comprender los estados emocionales, las intenciones y las impresiones de las otras personas. La componen un total de nueve ítems, y frases como las del siguiente tipo se integran en dicha escala: Me doy cuenta cuando alguien intenta esconder 
sus verdaderos sentimientos (item33); Entender cómo se siente otra persona es algo muy fácil para mí (item27).

c) Estrés empático. Implica la capacidad de compartir las emociones negativas de otra persona, es decir, de sintonizar emocionalmente con esta, ser permeable. Integra un total de ocho ítems y lo identificamos en frases como: A veces sufro más con las desgracias de las demáspersonas que ellas mismas (item 18); No puedo evitar llorar con los testimonios de personas desconocidas (item23).

d) Alegría empática. Remite a la capacidad de compartir las emociones positivas de otra persona y lo constituyen un total de ocho ítems. Por ejemplo: Cuando a alguien le sucede algo bueno siento alegría (item16); Me siento bien si las demás personas se divierten (item2).

Tras la obtención de las puntuaciones directas, se consulta en el manual el baremo adecuado, donde se ubica la puntuación $T$ y el percentil correspondiente. La baremación del instrumento fue construida a partir de una muestra de población adulta, con la generación de tablas, tanto generales como por sexo, y se expresa en puntuaciones percentiles y en puntuaciones transformadas $T$.

El análisis de los datos recabados mediante el TECA se realizó según procedimientos cuantitativos, se empleó el programa PSPP para el procesamiento y análisis de respuestas obtenidas, que fueron previamente codificadas.

\section{Principales resultados}

Entre los resultados se encontró que, desde una perspectiva global, el grupo de estudiantes de psicopedagogía evidencian niveles bajos de empatía (percentil 43), el cual es un nivel óptimo (situado entre los percentiles 7 y 93). Específicamente, se hallaron puntuaciones de nivel medio para la escala cognitiva (adopción de perspectivas y comprensión emocional) y puntuaciones bajas para las escalas afectivas (estrés empático y alegría empática). Para las personas profesionales de la educación, los niveles óptimos se encuentran entre los percentiles 69 y 93 para las escalas cognitivas y entre los percentiles 7 y 93 para las escalas afectivas (LópezPérez et al., 2008). Así, para este grupo de profesionales se consideran adecuados los niveles altos de empatía cognitiva y bajos de empatía afectiva.

TABLA 1 .

Media y desvío estándar sobre puntuaciones directas y percentiles para cada una de las escalas del TECA $(n=41)$.

\begin{tabular}{|c|c|c|c|c|c|c|}
\hline \multirow[t]{2}{*}{ Escalas } & \multirow[t]{2}{*}{$\mathrm{N}$} & \multicolumn{2}{|c|}{$\begin{array}{l}\text { Puntuaciones } \\
\text { Directas }\end{array}$} & \multirow[t]{2}{*}{ Pc } & \multirow[t]{2}{*}{ Puntuación T } & \multirow[t]{2}{*}{ Nivel } \\
\hline & & Media & Dt & & & \\
\hline Puntuación total & 41 & 107,61 & 7,88 & 25 & 43 & Baja \\
\hline Adopción de perspectivas & 41 & 26,61 & 3,60 & 35 & 46 & Media \\
\hline Comprensión emocional & 41 & 29,56 & 2,99 & 40 & 47 & Media \\
\hline Estrés empático & 41 & 21,68 & 3,67 & 25 & 43 & Baja \\
\hline Alegría empática & 41 & 29,76 & 2,24 & 25 & 43 & Baja \\
\hline
\end{tabular}

Fuente: Elaboración propia

En la Tabla 1 se sistematizan los valores obtenidos en la perspectiva global y en cada una de las escalas. Se mencionan los resultados relativos a las puntuaciones Media sobre las puntuaciones directas y los percentiles correspondientes. 


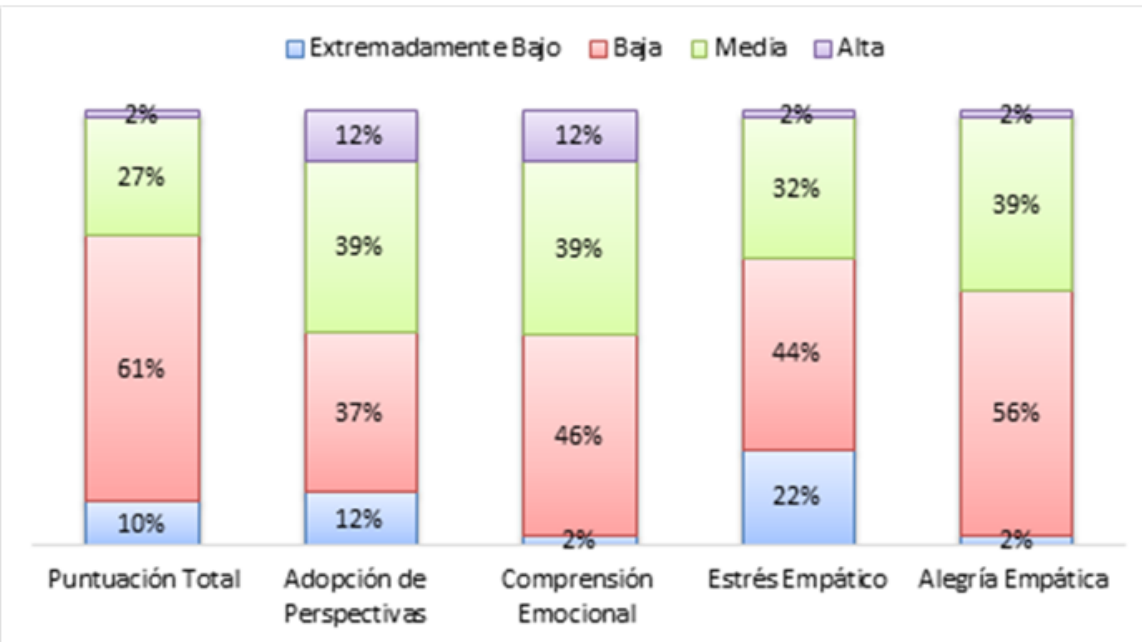

FIGURA 1.

Porcentaje de estudiantes representado por los diferentes niveles de empatía $(\mathrm{n}=41)$ Fuente: elaboración propia

La Figura 1 sistematiza el porcentaje de estudiantes y el nivel con el que se perciben con capacidad empática, tanto en su dimensión global como en sus cuatro escalas específicas: adopción de perspectiva, comprensión emocional, estrés empático y alegría empática. Como se puede observar, en general, la mayoría de quienes participaron de esta investigación obtuvieron puntuaciones bajas para Empatía Global. Específicamente, el $61 \%$ de participantes ha mostrado un nivel de empatía bajo, pero un valor extremo que llama la atención refiere a un $10 \%$ que se percibe con una puntuación en empatía extremadamente baja. A continuación, se precisa la interpretación de los resultados obtenidos al interior de cada una de las dimensiones de acuerdo con los planteamientos de las personas autoras del test (López-Pérez et al., 2008).

\subsection{Resultados relativos a la dimensión Empatía Cognitiva}

La escala Empatía Cognitiva está integrada -como ya se mencionó- por dos dimensiones o subescalas: adopción de perspectiva (AP) y comprensión empática (CE). De acuerdo con los resultados, en ambas dimensiones de esta escala, los principales valores se concentran en un nivel medio, tanto en AP como en $\mathrm{CE}$-esto es $3 \%$, respectivamente. Es importante atender a estos resultados en los contextos de formación profesional -como la universidad-, ya que es fundamental promover el desarrollo de niveles altos de empatía cognitiva para cualquier profesión. Particularmente, es esencial el desarrollo de éste tipo de empatía para el ejercicio de profesiones como psicopedagogía que requieren de una sensibilidad particular para comprender las necesidades de la otra persona, ya sea de sus pacientes -en caso de que se desempeñe en un contexto clínico-, de su alumnado -en caso de desempeñarse en docencia-, de docentes u otras personas que conforman el sistema educativos -si se desempeña en tareas de asesoramiento o capacitación pedagógica-, o bien, en el ámbito de la investigación científica. Poder empatizar desde lo cognitivo con las otras personas ayudará a profesionales en psicopedagogía a potenciar la calidad de sus servicios, al ofrecer una respuesta o intervención profesional más adecuada a la necesidad de cada situación, contexto, persona o grupo de personas.

Siguiendo con los resultados obtenidos para esta escala de empatía cognitiva, y según un orden decreciente de los porcentajes obtenidos, llama la atención que gran parte de las personas participantes de este estudio obtuvieron puntajes bajos y extremadamente bajos. Específicamente, un $37 \%$ obtuvo puntajes bajos en AP 
mientras que un $12 \%$ logró valores extremadamente bajos; asimismo, un $46 \%$ obtuvo puntuaciones bajas en CE y un $2 \%$ fueron extremadamente bajas.

Según los autores del TECA, las personas con puntuaciones bajas en AP suelen caracterizarse por tener un pensamiento menos flexible y, en tal sentido, pueden experimentar mayores dificultades para comprender el modo en que el otra piensa y lo que necesita. Una identidad profesional caracterizada por cierta rigidez mental supondría un cierto obstáculo en la fluidez de la comunicación y en relaciones establecidas con las demás personas. Consecuentemente, de acuerdo con lo expresado, una puntuación extremadamente baja en AP y baja en CE está asociado con importantes déficits en las habilidades de relación y comunicación con otras personas, debido a un estilo de pensamiento exageradamente rígido y focalizado en la perspectiva propia; generalmente manifiestan dificultades en su capacidad para establecer vínculos con las demás personas y mantener relaciones interpersonales de calidad. A estas personas, les cuesta comprender lo que el otra siente $y$, en tal sentido, es complicado para ellas entender lo que la otra necesita para estar mejor. Puntuaciones extremadamente bajas en CE -obtenidas por un $2 \%$ de la muestra-, reflejan una identidad profesional con problemas en las habilidades de relación con las demás personas, y dificultades emocionales a nivel interpersonal; esto es dificultad para efectuar una lectura emocional del comportamiento verbal y no verbal de otros y otras, lo que dificultaría detectar cuándo las demás personas experimentan emociones, tanto negativas como positivas.

\subsection{Resultados relativos a la dimensión Empatía Afectiva}

La escala empatía afectiva, como ya se hizo alusión, está conformada por dos dimensiones: estrés empático (EE) y alegría empática (AE). De acuerdo con los resultados de la investigación, en ambas dimensiones de esta escala, la mayoría de estudiantes se perciben con un nivel bajo -el $44 \%$ y $56 \%$, respectivamente-, y una parte de estudiantes se percibe con un nivel extremadamente bajo en la escala de EE (22\%). A diferencia de la escala antes descrita, y en íntima relación con el contexto que se está evaluando -futuras personas profesionales en psicopedagogía- es importante desarrollar esta capacidad de sintonizar con el otro o la otra, pero en niveles medio-bajos. Puntuaciones demasiado altas apuntan a una sobre implicación en los problemas ajenos -ya sean pacientes, estudiantes, docentes, dependiendo del ámbito donde se ejerza la profesión- o dejar en segundo plano la realización personal, lo que dificultaría la objetividad profesional, además de interferir negativamente en el ajuste emocional y, por tanto, perjudicar su salud psicológica. Por este motivo es importante atender a estos resultados en los contextos de formación profesional, promover identidades profesionales con un desarrollo de niveles intermedios de empatía afectiva.

Sin embargo, las personas que obtienen puntuaciones extremadamente bajas, por ejemplo, en EE -un $22 \%$ del total del grupo en estudio-, no se conmueven fácilmente, son poco emotivas, emocionalmente distantes y encuentran grandes dificultades en distinguir sus necesidades y emociones de las demás personas. Se caracterizan por una frialdad emocional excesiva, es decir, grandes dificultades para sentirse conmovidas por lo que sucede a otra persona y su red social suele ser de menor calidad que la de las personas con puntuaciones altas. En relación con la escala $\mathrm{AE}$, un pequeño grupo se percibe con un nivel extremadamente bajo (2\%). Puntuaciones bajas en esta escala denotan una menor tendencia a compartir las emociones positivas y manifiestan indiferencia ante los acontecimientos positivos que le sucede a las demás personas.

En el caso de los profesionales de la educación, se considera positivo manejar niveles medio-bajos de estrés empático y alegría empática, debido a que implica una distancia emocional óptima de las relaciones interpersonales y, a su vez, un manejo adecuado del estrés que evita manifestar una empatía excesiva hacia los problemas ajenos (pacientes, alumnos, etc.). Estos niveles suponen no involucrarse de manera exagerada en emociones tanto positivas como negativas de las demás personas. 


\subsection{Discusión de los resultados}

Las respuestas que proporcionó el estudiantado al test para avanzar hacia los objetivos referidos, señalan fortalezas y debilidades para este grupo, en particular en lo que respecta a su competencia empática. En cuanto a las fortalezas, los resultados hallados sugieren un grupo que, en general, se ha identificado con una capacidad empática cognitiva media y una capacidad empática afectiva baja -según las puntuaciones en cada una de las escalas al interior de las dimensiones-. Estos niveles son recomendados teóricamente, ya que en profesiones vinculadas con el contexto educativo es importante tener aptitudes relacionadas con comprender las necesidades del paciente o del alumnado, pero, al mismo tiempo, mantener cierta distancia con sus problemas y circunstancias para no perder objetividad profesional. Es considerado positivo que psicopedagogos y psicopedagogas mantengan puntajes bajos de empatía afectiva, esto es mantener una distancia emocional en sus relaciones, sin llegar al extremo alto o bajo.

Respecto de las posibles debilidades de este grupo de estudiantes en particular, llama la atención el subgrupo que se percibe con un grado de capacidad empática -tanto a nivel global como al interior de cada una de sus escalas- extremadamente bajo. Considerando los diferentes contextos donde se insertarían laboralmente las futuras personas profesionales, es tema de gran relevancia generar oportunidades que contribuyan a incrementar dicha competencia. Como fue mencionado anteriormente, niveles extremadamente bajos pueden interpretarse como déficits en las habilidades de relación y comunicación con otras personas por presentar un pensamiento rígido, dificultades emocionales a nivel interpersonal, frialdad emocional excesiva e indiferencia ante lo que les sucede a los demás. Estas cualidades pueden devenir, por ejemplo, en problemas a la hora del trabajo interdisciplinario - por la falta de comprensión sobre lo que necesitan quienes se acercan en busca de ayuda.

\section{SENDEROS PROMISORIOS DE ACTUACIÓN Y FUTURA INVESTIGACIÓN}

Respecto de los hallazgos presentados, surgen los siguientes interrogantes, ¿qué se puede hacer, como personas formadoras, para promover el desarrollo de empatía como competencia clave para el desempeño de la profesión?, ¿es posible ayudar al estudiantado a reparar sobre estos aspectos y promover su desarrollo a partir de la autovaloración? Si se considera que es importante generar oportunidades que ayuden al estudiantado a reflexionar sobre sus competencias socioemocionales, entonces se podrían sumar las siguientes preguntas, ¿qué oportunidades para poner en práctica estas competencias se proporcionan desde la universidad, entendida como una comunidad educativa? ¿Qué posibilidades concretas se generan desde los contextos de aprendizaje?, ¿Qué oportunidades se proporcionan desde las propuestas curriculares para contribuir a desarrollar empatía?

Con el fin de enriquecer los procesos de construcción de identidades profesionales integrales, desde las prácticas pedagógicas y desde la investigación educativa, se podrían considerar al menos tres senderos promisorios de actuación que podrían sugerir, a su vez, líneas de investigación: primero, promover oportunidades para el autoconocimiento; segundo, promover procesos de feedback orientados a destacar cualidades de la persona en situación de aprendizaje, más que aquellos enfocados en el resultado o logro obtenido; tercero, generar contextos ricos en oportunidades para la interacción social.

Respecto del primer sendero anticipado - promover oportunidades para el autoconocimiento-, se entiende que si se amplían las oportunidades que permitan a las personas estudiantes reflexionar sobre sí mismas, no sólo en su rol de estudiante, sino también como compañeras, amigas, hijas hermanas, etc., se enriquecerán sus posibilidades de re-conocerse en sus fortalezas, debilidades, sus metas, valores, recursos personales e incluso situacionales, y pensar en puntos de partida hacia mejoras en el desempeño de los diferentes roles. Con base en la investigación consultada sobre competencias socio-emocionales, un mayor 
autoconocimiento facilitaría el desarrollo de competencias interpersonales como la empatía (Goleman, 2013; Paoloni, 2019). Es aquí donde el personal docente se convierte en una pieza fundamental para contribuir a formar profesionales empoderados y empoderadas con estas capacidades. Cuando el contexto se entiende como una red y las acciones se estudian como acontecimientos particulares, los vínculos entre la persona, las demandas de la tarea, los propósitos de la acción y las herramientas materiales o simbólicas disponibles, pasan a ser objeto de mayor interés (Rinaudo, 2014). Cuestiones intrapersonales e interpersonales forman parte de cualquier contexto, y es importante esclarecerlas, hacerlas conscientes, reflexionar sobre ellas para poder utilizarlas como medio para seguir creciendo como persona y como profesional. En este marco, podrían sugerirse estudios de diseño (Rinaudo y Donolo, 2010) que acompañen estos procesos de enseñanza y de aprendizaje. Esto supone a personas investigadoras y docentes unidas por la intención de generar, ampliar o profundizar teoría existente, y complejizar las herramientas metodológicas con que se cuenta.

En cuanto al segundo sendero sugerido para la mejora de prácticas pedagógicas y de investigación educativa comprometidas con el desarrollo de empatía en el estudiantado, fueron propuestos los procesos de feedback. Desde perspectivas socioculturales amplias, los procesos de feedback en educación trascienden en mucho a los tradicionalmente considerados solo en relación con el desempeño del alumnado, en una tarea académica específica (Nicol y Macfarlance-Dick, 2004; Paoloni y Rinaudo, 2014). Así, se promueve la importancia de implementar un tipo particular de feedback que permita al estudiantado recibir información sobre rasgos de su actuación, no directamente vinculados con el resultado obtenido. Los autores lo llaman feedback relativo a aspectos personales. De tal modo, reconocer el valor del esfuerzo o de la perseverancia respecto de una actuación académica es mostrarle al estudiantado como en un espejo la capacidad-como docentes- de empatizar con ellos. Esta experiencia, que en el marco de estudios empíricos realizados resultó sumamente gratificante para el estudiantado (Paoloni y Rinaudo, 2014), posiblemente tenga el potencial de convertirse en una experiencia vicaria sobre empatizar con los demás. En función de lo expuesto, los procesos de feedback deben considerarse como herramienta inherente a las prácticas de enseñanza y de aprendizaje en diversas direcciones. Desde los procesos de formación docente sería enriquecedor invitar a las personas docentes a habilitar en sus clases instancias para la autoevaluación, la estimulación del diálogo entre el profesorado y pares respecto a los procesos de aprendizaje, la promoción de oportunidades para transformar las producciones, el desarrollo de creencias motivacionales positivas en el alumnado, así como la posibilidad de generar información que el personal docente pueda usar para mejorar sus prácticas de enseñanza.

Finalmente, un tercer sendero es generar contextos ricos en oportunidades para la interacción social, a modo de contribuir al desarrollo de la empatía como competencia clave en estudiantes avanzados de educación. Se plantea que si se generan contextos educativos donde primen los procesos de coconstrucción, es decir, entornos que den lugar a las relaciones interpersonales entre sus integrantes, donde se brinden “oportunidades para la colaboración entre pares" (Rinaudo, 2014, p. 192), se amplían las posibilidades de ejercer empatía. Se destaca el valor de capacitar a docentes para promover tareas auténticas y problemas genuinos, en entornos que den lugar a un aprendizaje fundamentalmente colaborativo y con sentido, caracterizado por la participación, la implicancia legítima y el compromiso sostenido de cada uno de sus integrantes, que se constituyen en rasgos identitarios y en espacios para el ejercicio de competencias socioemocionales, como lo es la empatía para futuros desempeños profesionales.

\section{Consideraciones Finales}

En este trabajo se propuso conocer más acerca de las percepciones de estudiantes de nivel avanzado de educación y sobre su capacidad para empatizar. Específicamente, se aspiró a identificar las percepciones que un grupo avanzado de estudiantes de la Licenciatura en Psicopedagogía tenían en relación con las dimensiones de empatía afectiva y cognitiva. Y a partir de allí generar reflexiones dirigidas a potenciar el diseño de contextos de aprendizaje que promuevan en el estudiantado mejoras en sus posibilidades para 
identificar y hacer uso inteligente de los recursos personales con que cuentan en su formación integral profesional.

Se partió de la importancia de considerar a la empatía como una competencia transversal clave para la construcción de las identidades integrales de estas futuras personas profesionales de la educación; el estudio se centró en la formación profesional de Licenciatura en Psicopedagogía. En profesiones vinculadas con el contexto educativo es importante tener aptitudes relacionadas con comprender las necesidades de pacientes o estudiantes, pero, al mismo tiempo, mantener cierta distancia con sus problemas y circunstancias para no perder objetividad profesional. Esto es poder empatizar desde lo cognitivo con el otro, lo cual ayudará a las personas profesionales en psicopedagogía a potenciar la calidad de sus servicios con intervenciones más adecuadas a la necesidad y acordes a la demanda, pero con una distancia emocional óptima de las relaciones interpersonales. Por este motivo, es importante fundar contextos instructivos potentes en sus posibilidades de promover procesos de coconstrucción de identidades profesionales integrales, donde se identifiquen los niveles de desarrollo de las competencias transversales -empatía en este caso- del estudiantado y trabajar sobre ello.

Para finalizar, se destacan posibles limitaciones y fortalezas del estudio aquí presentado. Una limitación tiene que ver con la posibilidad de generalización. Al respecto, cabe aclarar que nunca fue objetivo generalizar los resultados obtenidos, ya que no sería correcto metodológicamente ni tampoco conveniente como aspiración en sí misma. De hecho, se está valorando ampliar la muestra para validar el instrumento en población argentina. En cuanto a las fortalezas de este trabajo, se destaca la potencialidad y alcance de sus resultados. En este marco, se invita a pensar en la inclusión de la enseñanza de competencias socioemocionales en los diferentes planes de estudio. A su vez, se torna un desafío compartido y que, en este sentido, las alternativas propuestas como líneas de acción en este trabajo, pueden resultar de interés para otras personas docentes, autoridades, organismos, instituciones o personas investigadoras educacionales interesadas en mejorar la calidad de la educación para que responda más convenientemente a las nuevas demandas sociales.

En definitiva, los aportes de este estudio, al campo de la psicopedagogía y a las futuras líneas de investigación que pueden derivarse, tienen el potencial de promover una comprensión más acabada y profunda acerca de contextos de aprendizajes comprometidos con la construcción de identidades profesionales integrales. Si la identidad profesional es una construcción compartida entre persona y contexto, entonces se puede pensar en oportunidades de aprendizaje que ayuden a las futuras personas profesionales a desarrollar empatía como una competencia que les permitirá un mejor desempeño de su rol. Lo que en este trabajo sugiere a partir de los hallazgos obtenidos son algunas alternativas de trabajo, aunque, como toda alternativa, conlleva el germen de un devenir, una posibilidad de llevar al destino anhelado.

\section{Referencias Bibliográficas}

Aisenson, D., Monedero, F., Batlle, S., Legaspi, L., Aisenson, G., Vidondo, M., Nicotra, D., Valenzuela, V., Davison, S. y Alonso, D. (2005). Representaciones de estudiantes y graduados recientes sobre la carrera y la profesión del psicólogo. Anuario de Investigaciones, 12(1), 35-42. Recuperado de https://www.redalyc.org/pdf/3691/36913 9941002.pdf

Aristulle, P. D. C. y Paoloni, P. V. (2019). Habilidades socioemocionales en las comunidades educativas: aportes para la formación integral de los y las docentes. Revista Educación, 43(2), 49-64. doi: https://doi.org/10.15517/rev edu.v43i2.28643

Ávila-Alarcón, V., Carrasco-Alfaro, C., Osorio-Cerda, L., Calzadilla-Núñez, A. y Díaz-Narváez, V. P. (2020). Estudio trasversal de empatía con el paciente en estudiantes de kinesiología. Educación Médica Superior, 34(2). Recuperado de https://www.medigraphic.com/pdfs/educacion/cem-2020/cem202h.pdf 
Bar-On, R. (2000). Emotional and social intelligence: insights from emotional quotient inventory. En R. Bar-On y J. D. Parker (Eds.), The handbook of emotional intelligence: theory, development, assessment and application at home, school and in the workplace (pp. 363-388). San Francisco, CA: Jossey-Bass.

Bar-on, R. (2006). The Bar-On Model of Emotional-Social Intelligence. Psicothema, 18(13-25). Recuperado de http ://www.psicothema.com/pdf/3271.pdf

Bar-On, R. (2012). Inteligencia Emocional y felicidad. Entrevista a Reuven Bar-On. Recuperado de http://www.yout ube.com/watch?v=h5jGibf4wyE

Baron-Cohen, S. y Wheelwright, S. (2004). The Empathy Quotient: An Investigation of Adults with Asperger Syndrome or High Functioning Autism, and Normal Sex Differences. Journal of Autism and Developmental Disorders, 34(2), 163-175. doi: https://doi.org/10.1023/B:JADD.0000022607.19833.00

Bisquerra, R. (2003). Educación emocional y competencias básicas para la vida. Revista de Investigación Educativa, 21(1), 7-43. Recuperado de https://revistas.um.es/rie/article/download/99071/94661/0

Bong, M. y Skaalvik, E. M. (2003). Academic Self-Concept and Self-Efficacy: How Different Are They Really? Educational Psychology Review, 15(1), 1-40. doi: https://doi.org/1040-726X/03/0300-0001/0

Bryant, B. K. (1982). An Index of Empathy for Children and Adolescents. Child Development, 53, 413-425. doi: ht tps://doi.org/10.2307/1128984

Chiecher, A. C., Paoloni, P. V. y Ficco, C. R. (2014). Ingreso a la Universidad en modalidad a distancia. El papel de aspectos motivacionales y cognitivos en la configuración de logros académicos. RED. Revista de Educación a Distancia, 43, 1-25. Recuperado de https://www.um.es/ead/red/43/chiecher_et_al.pdf

Colby, A., Ehrlich, T., Beaumont, E. y Stephens, J. (2003). Educating citizens: Preparing America's undergraduates for lives of moral and civic responsibility (6). San Francisco: Jossey-Bass.

Czapski, M. y Lassi, M. (2020). Empatía en estudiantes de medicina procedentes de Brasil y Argentina: Diferencias por sexo y nivel en la carrera. Subjetividad y procesos cognitivos, 24(1), 22-40. Recuperado de https://bit.ly/3uiHdmq

Davis, M. H. (1983). Measuring individual differences in empathy: Evidence for a multidimensional approach.Journal of Personality and Social Psychology, 44(1), 113-126. doi: https://doi.org/10.1037/0022-3514.44.1.113

Davis, M. H. (1996). Empathy. A social psychological approach. Routledge. doi: https://doi.org/10.1007/978-981-10 -5541-6_1

Decety, J.y Jackson, P. L. (2004). The functional architecture of human empathy. Behavioral and cognitive neuroscience reviews, 3(2), 71-100. doi: https://doi.org/10.1177/1534582304267187

Elliot, P. (1975). Sociología de las profesiones. Madrid: Tecnos.

Gaeta, M. L. (2014). La autorregulación del aprendizaje y su promoción en el contexto del aula. En P. V. Paoloni, M. C. Rinaudo, y A. González-Fernández (Comps.), Cuestiones en Psicología Educacional Perspectivas teóricas, metodológicas y estudios de campo (pp. 33-57). Sociedad Latina de Comunicación Social (SLCS). http://www.c uadernosartesanos.org/2014/cde01.pdf

Gaeta, M. L. y Martínez-Otero, V. (2017).Las competencias emocionales en la educación formal. Reflexiones y experiencias de investigación en diferentes contextos educativos. Ciudad de México: Universidad Popular Autónoma del Estado de Puebla, Colofón.

Gardner, H. (1998). Inteligencias Múltiples. La teoría en la práctica. España: Paidós Ibérica.

Gerdes, K. E. y Segal, E. A. (2009). A Social Work Model of Empathy. Advances in Social Work, 10(2), 114-127. doi: https://doi.org/10.18060/235

Goleman, D. (2000). La inteligencia emocional. Por qué es más importante que el cociente intelectual. Buenos Aires: Vergara Ediciones.

Goleman, D. (2007). La inteligencia emocional. Buenos Aires: Vergara.

Goleman, D. (2013). La inteligencia emocional en la empresa. Buenos Aires: Zeta Bolsillo.

Guilera, T., Batalla, I. y Soler-González, J. (2020). Shadowing patients: Experiencing empathy in medical students. Educación Medica, 21(2), 112-117. doi: https://doi.org/10.1016/j.edumed.2018.06.006 
Hernández-Sampieri, R. (2018). Definición del alcance de la investigación en la ruta cuantitativa: exploratorio, descriptivo, correlacional o explicativo. En R. Hernández-Sampieri y C. P. Mendoza-Torres (Eds.), Metodología de la investigación: las tres rutas cuantitativa, cualitativa y mixta (2da ed., Vol. 1, pp. 104-120). McGraw-Hill. Recuperado de http://www.mhhe.com/latam/sampieri_mile

Hernández, L., Martín, C. I., Lorite, G. y Granados, P. (2018). Rendimiento, motivación y satisfacción académica, ¿una relación de tres? ReiDoCrea: Revista electrónica de investigación Docencia Creativa, 7(2014), 92-97. doi: h ttps://doi.org/10.30827/Digibug.49829

Hoffman, M. L. (1982). The measurement of Empathy. En C. E. Izard (Ed.), Measuring Emotions in Infants and Children (pp. 279-296). Cambridge - Massachusetts: Cambridge University Press.

Hoffman, M. L. (2001). Empathy and moral development: Implications for caring and justice. Cambridge. Massachusetts: Cambridge University Press.

Hogan, R. (1969). Development of an empathy scale. Journal of Consulting and Clinical Psychology, 33(3), 307-316. doi: https://doi.org/10.1037/h0027580

Huarcaya-Victoria, J., Cano-Uría, B., Villanueva-Ruska, A. y de la Cruz-Oré, J. (2019). Evaluation of the levels of medical empathy in medical residents of a general hospital in Peru. Educación Medica, 20, 59-66. doi: https:// doi.org/10.1016/j.edumed.2018.04.004

Järvelä, S. y Niemivirta, M. (2001). Motivation in context: Challenges and possibilities in studying the role of motivation in new pedagogical cultures. En S. Volet y S. Järvelä (Eds.), Advances in learning and instruction series. Motivation in learning contexts: Theoretical advances and methodological implications (pp. 105-127). Londres: Pergamon Press.

Lasky, S. (2005). A sociocultural approach to understanding teacher identity, agency and professional vulnerability in a context of secondary school reform. Teaching and Teacher Education, 21(8), 899-916. doi: https://doi.org/1 0.1016/j.tate.2005.06.003

López-Pérez, B., Fernández-Pinto, I. y Abad García, F. J. (2008). TECA: test de empatía cognitiva y afectiva. TEA Ediciones.

Marsh, H. W. (2014). Academic self-concept: Theory, measurement, and research. Psychological Perspectives on the Self, 4, 71-110.

Martínez-Otero, V. (2017). La trascendencia de la empatía en la Educación: conclusiones a partir de un estudio con alumnos universitarios. En M. L. Gaeta y V. Martínez-Otero (Coords.), Las competencias emocionales en la educación formal. Reflexiones y experiencias de investigación en diferentes contextos educativos (p.p. 105-118). Ciudad de México: Universidad Popular Autónoma del Estado de Puebla, Colofón.

Mayer, J. D. y Salovey, P. (1997). What is emotional intelligence? En P. Salovey y D. J. Sluyter (Eds.), Emotional development and emotional intelligence: Educational implications (pp. 3-31). New York, NY: Basic Books.

Medina, P. (2020). La inteligencia emocional y su relación con el apoyo social y el autoconcepto como factores que influyen en el rendimiento académico de estudiantes de los niveles primario, secundario y superior. Revista Cientifica Arbitrada de la Fundación MenteClara, 5, 1-14. doi: https://doi.org/10.32351/rca.v5.203

Mehrabian, A. (1976). Questionnaire Measures of Affiliative Tendency and Sensitivity to Rejection. Psychological Reports, 38(1), 199-209. doi: https://doi.org/10.2466/pr0.1976.38.1.199

Mehrabian, A. y Epstein, N. (1972). A measure of emotional empathy1. Journal of Personality, 40(4), 525-543. doi: h ttps://doi.org/10.1111/j.1467-6494.1972.tb00078.x

Miller, P. A. y Eisenberg, N. (1988). The relation of empathy to aggressive and externalizing/antisocial behavior. Psychological Bulletin, 103(3), 324-344. doi: https://doi.org/10.1037/0033-2909.103.3.324

Nicol, D. y Macfarlance-Dick, D. (2004). Rethinking Formative Assessment in HE: a theoretical model and seven principles of good feedback practice. En C. Juwah, D. MacFarlane-Dick, B. Matthew, D. Nicol, D. Ross, y B. Smith (Comps.), Enhancing student learning though effective formative feedback (pp. 3-14).Estados Unidos: The Higher Education Academy. Recuperado de https://bit.ly/3eRcolx 
Oyarzún-Yáñez, R. y Valdés-León, G. (2020). Emociones, motivación y rendimiento académico: una propuesta para el desarrollo de habilidades orales en ingeniería desde la neuroeducación. Centro Sur. Social Science Journal, 4(2), 252-265. doi: https://doi.org/10.37955/cs.v4i2.80

Pacheco-Hernández, P. (2016). Positividad y Negatividad emocional: influencia en los procesos de aprendizajes. Estudios pedagógicos (Valdivia), 42(1), 187-207. doi: https://doi.org/10.4067/S0718-07052016000100012

Paoloni, P. V. (2014). Percepciones autorreferenciales de ingresantes en ingeniería. Aportes para la configuración de situaciones de logro. Libro de actas de VI Congreso Internacional de Investigación y Práctica Profesional en Psicologia XXI Jornadas de Investigación Décimo Encuentro de Investigadores en Psicología del MERCOSUR. Facultad de Psicologia - Universidad de Buenos Aires, pp. 202-206. Recuperado de https://www.aacademica.or $\mathrm{g} / 000-035 / 402$

Paoloni, P. V. (2015). Abandono y permanencia en Carreras de Ingeniería. Un estudio orientado a detectar factores de riesgo y fortalezas entre los ingresantes. En M. Panaia (Coord.), Universidades en cambio: igeneralistas o profesionalizantes? (pp. 135-158). Buenos Aires: Miño y Dávila.

Paoloni, P. V. (2018). [Reseña] Las competencias emocionales en la educación formal. Reflexiones y experiencias de investigación en diferentes contextos educativos. Revista Iberoamericana de Educación, 77(2), 179-183. doi: ht tps://doi.org/10.35362/rie7723157

Paoloni, P. V. (2019). Competencias socioemocionales ayer, hoy... ¿y mañana? En P. V. Paoloni, M. C. Rinaudo y R. B. Martín (Comps.), Yo, tú... ellos y nosotros. Competencias socioemocionales en la construcción de identidades profesionales (pp. 103-134). Córdoba: Editorial Brujas.

Paoloni, P. V. y Rinaudo, M. C. (2014). Los procesos de feedback desde una perspectiva multidimensional. Un estudio orientado a promover autorregulación en estudiantes universitarios. En P. V. Paoloni, M. C. Rinaudo, y A. González-Fernández (Comps.), Cuestiones en Psicología Educacional:perspectivas teóricas, metodológicas yestudios de campo (pp. 287-324). España: Sociedad Latina de Comunicación Social (SLCS). Recuperado de http://ww w.cuadernosartesanos.org/2014/cde01.pdf

Paoloni, P. V.y Rinaudo, M. C. (2015). Dinámica emocional en contextos de evaluación. Un estudio longitudinal con alumnos de ingeniería. Revista Novedades Educativas, 288/289, 30-35.

Paoloni, P. V., Chiecher, A. C. y Martín, R. B. (2015). Percepciones autorreferenciales y expectativas por contextos de aprendizaje. Un estudio orientado a prevenir el abandono de ingresantes en carreras de ingeniería. Acta de la Quinta Conferencia Latinoamericana sobre el Abandono en la Educación Superior (CLABES) Universidad de Talca. Recuperado de https://revistas.utp.ac.pa/index.php/clabes/article/view/1151

Paoloni, P. V., Rinaudo, M. C., Chiecher, A. C. y Martín, R. B. (2017). La dimensión motivacional en la construcción de identidades profesionales. En A. Riccetti, A. C. Chiecher, y D. S. Donolo (Comps.), Pan, queso y ojotas. Estudios alternativos sobre el deporte. Cuadernos de Comunicación (pp. 337-375). España: Sociedad Latina de Comunicación Social (SLCS). Recuperado de http://www.cuadernosartesanos.org/2017/cde07.pdf

Pintrich, P. R. y Schunk, D. H. (1996). Motivation in education. New Jersey: Prentice Hall, Inc.

Repetto-Talavera, E. y Pérez-González, J. (2007). Formación en competencias socioemocionales a través de las prácticas en empresas. Revista Europea de Formación Profesional, 40, 92-112. Recuperado de https://dialnet.un irioja.es/descarga/articulo/2316242.pdf

Richaud, M. C. (2017). ¿La empatía es disposicional o social? La compleja relación entre la empatía y la prosocialidad. Seminario "Persona, mente y cerebro" - Proyecto "Cerebro y Persona" (2016-2019). Universidad Austral, 2000, 1-6. Recuperado de https://bit.ly/3pekqpM

Rinaudo, M. C. (2014). Estudios sobre los contextos de aprendizaje: arenas y fronteras. En P. V. Paoloni, M. C. Rinaudo, y A. González-Fernández (Comps.), Cuestiones en Psicología Educacional. Perspectivas teóricas, metodológicas y estudios de campo (pp. 163-205). España: Sociedad Latina de Comunicación Social (SLCS). Recuperado de http://www.cuadernosartesanos.org/2014/cde01.pdf

Rinaudo, M. C. (2019). El estudio de la identidad en el campo de la Psicología Educacional. En P. V. Paoloni, M. C. Rinaudo y R. B. Martín (Comps.), Yo, tú... ellos y nosotros. Competencias socioemocionales en la construcción de identidades profesionales (pp. 27-74). Córdoba: Editorial Brujas. 
Rinaudo, M. C. y Donolo, D. (2010). Estudios de diseño. Una perspectiva prometedora en la investigación educativa. RED. Revista de Educación a Distancia, 22, 1-29. Recuperado de https://revistas.um.es/red/article/view/1116 $31 / 105951$

Rinaudo, M. C. y Paoloni, P. V. (2015). Estudiantes universitarios. Rosas... cardos y ortigas en la construcción de identidades profesionales. Revista de Docencia Universitaria (REDU), 13(2), 73-90. doi: https://doi.org/10.49 95/redu.2015.5439

Ruiz-Baca, P. (2016). Propiedades Psicométricas del test de empatía cognitiva y afectiva en estudiantes no universitarios. Cátedra Villarreal Psicología, 1(1), 99-116. doi: http://dx.doi.org/10.24039/cv201311127

Ruiz-Gonzalez, E. (2017). Propiedades psicometricas del Test de Empatía Cognitiva y Afectiva (TECA) en población colombiana. Libro de Acta de XI Congreso Internacional de Investigación y Práctica Profesional en Psicología. XXVI Jornadas de Investigación. XV Encuentro de Investigadores en Psicologia del MERCOSUR. I Encuentro de Investigación de Terapia Ocupacional. I Encuentro de Musicoterapia. Buenos Aires: Facultad de PsicologíaUniversidad de Buenos Aires. Recuperado de https://www.aacademica.org/000-111/140

Ryan, R. M. y Deci, E. L. (2000). Intrinsic and Extrinsic Motivations: Classic Definitions and New Directions. Contemporary Educational Psychology, 25(1), 54-67. doi: https://doi.org/10.1006/ceps.1999.1020

Sevilla-Santo, D. E., Martín-Pavón, M. J., Sunza-Chan, S. P. y Druet-Domínguez, N. V. (2021). Autoconcepto, expectativas y sentido de vida: Sinergia que determina el aprendizaje. Revista Electrónica Educare, 25(1), 1-23. doi: https://doi.org/10.15359/ree.25-1.12

Sorolla, M. N., Álvarez, G. N. y Penna, F. (2019). Relación entre motivación de aprendizaje y progreso académico en estudiantes de las Facultades de Psicología y Ciencias Humanas ( UNSL ). RevID - Revista de Investigación y Disciplinas, 1, 87-102. Recuperado de https://www.evirtual.unsl.edu.ar/revistas/index.php/revid

Vallaeys, F., De la Cruz, C. y Sasia, P. M. (2009). Responsabilidad social universitaria. Manual deprimerospasos. Madrid: McGraw Hill Interamerica. Recuperado de https://bit.ly/2GSyQup

Vital, L. M., Gaeta, M. L. y Martínez-Otero, V. (2017). La empatía docente: su implicación en la relación maestro alumno en la educación inicial. En M. L. Gaeta y V. Martínez-Otero (Coords.), Las competencias emocionales en la educación formal. Reflexiones y experiencias de investigación en diferentes contextos educativos (pp. 19-38). Ciudad de México: Universidad Popular Autónoma del Estado de Puebla, Colofón.

Volet, S. (2011). Emerging Trends in Recent Research on Motivation in Learning contexts. En S. Volet y S. Järvelä (Eds.), Motivation in Learning Contexts. Theoretical Advances and Methodological Implications (pp. 319-334). Londres: Pergamon Press.

Zorza, J. P. y Beccaría, E. (2014). Relación entre empatía y regulación emocional en adolescentes. Acta de III Jornada sobre divulgación de investigación en Ciencias Sociales., 1-3. Recuperado de https://bit.ly/2GRETPU

\section{Notas}

[1] "Los estudios exploratorios sirven para preparar el terreno y generalmente anteceden a investigaciones con alcances descriptivos, correlacionales o explicativos" (Hernández-Sampieri, 2018, p. 106). Se está valorando ampliar la muestra para validar el instrumento en población argentina.

[2] Diferentes estudios comprueban las propiedades psicométricas del TECA. Entre otros, Ruiz-Baca (2016) trabajó con estudiantes de un Instituto Superior de Chepén (Perú). Sus resultados muestran una validez de constructo entre 0,352 a 0,484 -correspondientes a niveles bueno y muy bueno, respectivamente-. A su vez, el alfa de Cronbach demostró una confiabilidad de 0,814 . Otra investigación que evaluó las bondades técnicas del instrumento con población colombiana arrojó resultados muy satisfactorios, a saber: un alfa de Cronbach de 0,89, para el test total; 0,84 para la subescala Empatía Cognitiva y 0,94 para la subescala Empatía Afectiva (Ruiz-Gonzalez, 2017).

\section{INFORMACIÓN ADICIONAL}


Daiana Schlegel, et al. Percepciones del estudiantado argentino de Psicopedagogía en relación a su...

Cómo citar: Schlegel, D., Paoloni, P.V. y Gaeta, M.L. (2021). Percepciones del estudiantado argentino de Psicopedagogía en relación a su capacidad empática en la construcción de identidad profesional. Revista Educación, 45(2). Recuperado de http://doi.org/10.15517/revedu.v45i1.44467 